\title{
Metal halide perovskites for light-emitting diodes
}

Xiaoke Liu, Weidong Xu, Sai Bai, Yizheng Jin, Jianpu Wang, Richard H. Friend and Feng Gao

The self-archived postprint version of this journal article is available at Linköping University Institutional Repository (DiVA):

http://urn.kb.se/resolve?urn=urn:nbn:se:liu:diva-170571

N.B.: When citing this work, cite the original publication.

Liu, X., Xu, W., Bai, S., Jin, Y., Wang, J., Friend, R. H., Gao, F., (2020), Metal halide perovskites for light-emitting diodes, Nature Materials. https://doi.org/10.1038/s41563-020-0784-7

Original publication available at:

https://doi.org/10.1038/s41563-020-0784-7

Copyright: Nature Research

http://www.nature.com/ 


\section{Metal halide perovskites for light-emitting diodes}

Xiao-Ke Liu ${ }^{1 \dagger}$, Weidong Xü ${ }^{1 \dagger}$, Sai Bai ${ }^{1 \dagger}$, Yizheng Jin² ${ }^{2}$ Jianpu Wang ${ }^{3}$, Richard H. Friend ${ }^{4}$ and Feng $\mathrm{Gao}^{1 *}$

${ }^{1}$ Department of Physics, Chemistry and Biology (IFM), Linköping University, Linköping SE58183, Sweden.

${ }^{2}$ Center for Chemistry of High-Performance and Novel Materials, State Key Laboratory of Silicon Materials, and Department of Chemistry, Zhejiang University, 310027 Hangzhou, China.

${ }^{3}$ Key Laboratory of Flexible Electronics (KLOFE) \& Institute of Advanced Materials (IAM), Jiangsu National Synergetic Innovation Center for Advanced Materials (SICAM), Nanjing Tech University (NanjingTech), 30 South Puzhu Road, 211816 Nanjing, China.

${ }^{4}$ Cavendish Laboratory, J J Thomson Avenue, Cambridge CB3 0HE, UK.

†These authors contributed equally to this work.

*e-mail: feng.gao@liu.se 


\begin{abstract}
Metal halide perovskites have shown promising optoelectronic properties suitable for lightemitting applications. The development of perovskite light-emitting diodes (PeLEDs) has progressed rapidly over the past several years, reaching high external quantum efficiencies of over $20 \%$. In this Review, we focus on the key requirements for high-performance PeLEDs, highlight recent advances on materials and devices, and emphasize the importance of reliable characterizations of PeLEDs. We discuss possible approaches to improve the performance of blue and red PeLEDs, increase the long-term operational stability, and reduce toxicity hazards. We also provide an overview of the application space made possible by recent developments of high-efficiency PeLEDs.
\end{abstract}


Metal halide perovskites, which have led to great advances in photovoltaic devices, have also proved to be promising candidates for light-emitting diodes (LEDs) ${ }^{1}$. They have shown excellent optoelectronic properties suitable for LEDs, such as high photoluminescence quantum yields (PLQYs), widely tunable bandgap, narrow emission width, and high charge-carrier mobility ${ }^{2}$. Although early reports on perovskite LEDs (PeLEDs) date back to the $1990 \mathrm{~s}^{3,4}$, room-temperature PeLEDs were not demonstrated until 2014 . Since then, benefiting from established experience in both perovskite materials and solution-processed optoelectronic devices, the community has quickly boosted the external quantum efficiencies (EQEs) of PeLEDs to each more than $20 \%$ (Box 1) $\left(\right.$ refs $^{6-10}$ ).

The rapid development of PeLEDs could lead to a new generation of low-cost and highperformance LEDs for applications including displays, lighting and optical communications ${ }^{2,11,12}$. Compared with other emitters used in commercial devices, such as IIIV inorganic semiconductors, organic emitters and conventional colloidal quantum dots (QDs), perovskites have several promising characteristics. Specifically, perovskite emitters with high PLQYs can be straightforwardly fabricated from low-cost precursor solutions, potentially reducing manufacturing costs. Synthesis of colloidal perovskite nanocrystals (PNCs) is also simplified, as PNCs can reach near-unity PLQYs without delicate shell passivation, owing to their unique defect-tolerance nature ${ }^{13,14}$. Furthermore, the optoelectronic properties of perovskite emitters can be readily tailored by engineering composition and dimensionality, enabling continuously tunable light emission from violet to near-infrared (NIR) regions ${ }^{2,13}$. In addition, light emission from perovskites shows narrow linewidths $(<100 \mathrm{meV})$, resulting in high color purity: for example, the photoluminescence full width at half maximum (FWHM) is around 12, 20 and $40 \mathrm{~nm}$ for $\mathrm{CsPbCl}_{3}, \mathrm{CsPbBr}_{3}$ and $\mathrm{CsPbI}_{3} \mathrm{PNCs}_{\text {, respectively }}{ }^{15}$. The color gamut of displays made by PNCs can cover up to $140 \%$ of the National Television System 
Committee standard and is close to $100 \%$ of the new International Telecommunication Union Rec. 2020 standard, making them promising for ultra-high-definition displays ${ }^{16}$.

Yet the deployment of perovskite emitters in commercial devices presents barriers that need to be carefully addressed. We open this Review discussing strategies for achieving highly emissive perovskite emitters through effective control of both radiative and nonradiative recombination processes. We then focus on critical device prerequisites to achieve highefficiency PeLEDs, highlighting similarities and differences between the device engineering of PeLEDs and other LEDs, and emphasizing the importance of reliable device characterization for this emerging technology. We also overview specific challenges and possible solutions in realizing high-performance red and blue PeLEDs, improving operational stability and reducing the toxicity of these devices. We finally discuss future applications of perovskite emitters.

\section{Towards highly emissive metal halide perovskites}

From a material point of view, the prerequisite for achieving highly efficient LEDs is to use light emitters with high PLQYs. For any luminescent material, its PLQY is defined as the number of photons emitted divided by the number of photons absorbed, and can also be expressed by the ratio between its radiative recombination rates and the overall recombination rates:

$\mathrm{PLQY}=\frac{\sum R_{r}}{\sum R_{r}+\sum R_{n r}}$

where $R_{r}$ and $R_{n r}$ are the radiative and nonradiative recombination rates, respectively. Equation (1) indicates that the general principle for achieving a highly efficient light emitter is to enhance its radiative recombination rate(s) and to suppress the nonradiative recombination rate(s). In metal halide perovskites, the radiative recombination can be bimolecular or excitonic, whereas trap-assisted monomolecular and Auger recombination are nonradiative processes ${ }^{17,18}$. 
In this section, we discuss strategies for achieving highly efficient perovskite emitters through effective control over both the radiative and nonradiative recombination processes.

Enhancing the radiative bimolecular recombination rate. Three-dimensional (3D) perovskite emitters generally possess small exciton binding energies close to the room temperature thermal energy, indicating that the excitons in perovskites readily dissociate into free charge carriers ${ }^{18}$. Because bimolecular recombination rates are usually slow in typical 3D perovskites, free carriers can be easily captured by non-radiative recombination centres at low charge-carrier densities (as illustrated in Fig. 1a). At relatively high charge-carrier densities, traps are saturated and radiative recombination can be dominant. The charge-carrier recombination kinetics in 3D perovskites is strongly dependent on charge-carrier density, and can be described by Equation (2) ${ }^{18,19}$,

$-\frac{d N}{d t}=A N+B N^{2}+C N^{3}$

where $N$ is the charge-carrier density, $t$ is time, $A$ is the trap-assisted nonradiative monomolecular recombination coefficient, $B$ is the radiative bimolecular recombination coefficient and $C$ is the three-body Auger (nonradiative) recombination coefficient. The PLQYs of 3D perovskite emitters can hence be quantified by Equation (3):

$P L Q Y=\frac{B N}{A+B N+C N^{2}}$

Figure $1 \mathrm{~b}$ and $\mathrm{c}$ illustrate the excitation density dependent PLQYs of 3D perovskites with different recombination coefficients. These calculations indicate the importance of increasing $B$ (Fig. 1b) and decreasing $A$ values (Fig. 1c), so that the PLQYs can approach unity in a wide excitation density range relevant for practical LED operations.

Although bimolecular recombination rates in typical 3D perovskites are usually slow under the LED operating conditions ${ }^{19}$, they can be efficiently enhanced by spatially confining the charge carriers in perovskite emitters. Early work on room-temperature PeLEDs demonstrated a useful strategy for confining the charge carriers by using thin perovskite layers $(\sim 30 \mathrm{~nm})^{5}$. 
Similar confinement effects can also be obtained by decreasing the perovskite grain sizes; this has been obtained by constraining crystal growth through, for instance, engineering the precursor stoichiometry ${ }^{20}$, incorporating ammonium halides with large organic cations ${ }^{21}$ and embedding the perovskite grains in polymer matrixes ${ }^{22}$. In addition, confining one of the carriers through local variations in band edge energies can switch the decay to first order kinetics and give enhanced PLQYs, as reported for 'photodoping' in a mixed cation/anion system $^{23}$.

Using radiative excitonic recombination. In addition to enhancing radiative bimolecular recombination, another strategy to increase PLQYs is to make use of radiative monomolecular recombination pathways. This could be readily achieved through developing quantum-confined perovskites such as two-dimensional (2D) perovskites, quasi-2D perovskites or PNCs (Box 1). In contrast to free charge carriers generated in 3D perovskites, stable excitons can form in these quantum-confined perovskites at room temperature (as illustrated in Fig. 1d) due to their high exciton binding energies, up to hundreds of millielectronvolts ${ }^{17}$. This feature results in fast radiative monomolecular recombination rates, and hence recombination dynamics in this case are less dependent on charge carrier densities.

In quantum-confined perovskites, first-order radiative excitonic recombination competes with first-order nonradiative recombination through charge- or exciton-trapping and with second-order non-radiative Auger recombination of exciton-electron/hole or exciton-exciton annihilation ${ }^{24}$. Therefore, charge-carrier recombination kinetics and PLQYs can be described by Equation (4) and (5), respectively:

$-\frac{d N}{d t}=\left(k_{t r a p}+k\right) N+k_{2} N^{2}$

and

$$
P L Q Y=\frac{k}{\left(k_{\text {trap }}+k\right)+k_{2} N}
$$


where $k_{\text {trap }}, k$ and $k_{2}$ are rate constants of nonradiative monomolecular recombination, radiative monomolecular recombination, and Auger recombination, respectively. Accordingly, under low excitation densities where the second-order Auger process is negligible, the PLQY is equal to $\frac{k}{\left(k_{\text {trap }}+k\right)}$, which is independent of the excitation density (Fig. 1e). With finely controlled trap densities, first-order excitonic radiative recombination could outperform the nonradiative recombination, leading to high PLQYs even at low excitation densities (Fig. 1e). Indeed, nearunity PLQYs have been demonstrated in a range of PNCs, 2D and quasi-2D perovskites ${ }^{10,25}$.

We note that, in practical cases, the radiative processes of perovskite emitters might be more complicated. For example, in quasi-2D perovskites with large $n$ values and PNCs with crystal sizes close to exciton Bohr diameters, both excitonic and free-carrier recombination may contribute to the light emission and collectively determine the PLQYs. In addition, in mixed dimensional perovskites (mixtures of 2D/quasi-2D/3D phases), radiative recombination can happen at different phases, and there is usually energy transfer from the large bandgap phases to the low bandgap phase ${ }^{26,27}$. Furthermore, the presence of materials with excited-state energies lower than those of the perovskite emitters (such as additives in 3D perovskites, organic spacers in $2 \mathrm{D}$ or quasi-2D perovskites) can result in energy transfer from the perovskite emitters to these materials ${ }^{4,28,29}$, leading to lower PLQYs or emission from these lower-energy materials.

Suppressing trap-assisted nonradiative recombination. In addition to the above strategies on enhancing the radiative recombination rates, minimizing trap-assisted nonradiative recombination pathways is equally important for maximizing PLQYs of perovskite emitters. Trap states are usually associated with intrinsic point defects, such as vacancies, interstitials and anti-site occupations (Fig. 2a). So far, several types of point defects, including halide and A-site vacancies, $\mathrm{Pb}$-halide anti-sites, metallic $\mathrm{Pb}^{0}$, have been experimentally demonstrated to be associated with nonradiative trap states ${ }^{30,31}$. 
Eliminating the negative effects of trap states has been intensively investigated in perovskite PVs, and various useful defect passivation strategies have been developed ${ }^{30}$. The basic concept of defect passivation in perovskites is to heal the dangling bonds by employing passivation agents that provide extra coordination or ionic bonding to neutralize the charged defects, leading to annihilation of electronic trap states. Generally, useful passivation agents can be introduced by loading them in precursors for film deposition or colloidal synthesis of perovskites, or by performing post-treatment on the crystallized perovskites ${ }^{6,32}$. A wide range of defect passivation agents or ligands, including molecules containing Lewis acid/base moieties, positively/negatively charged organic components, and alkali metal ions, have been demonstrated to be efficient in reducing the defect density of perovskites, leading to increased PLQYs of perovskite emitters and improved performance of PeLEDs ${ }^{33}$.

Such studies have also been instrumental to understanding the role of chemical structures of passivation agents on their effectiveness. For instance, the successful passivation of halide vacancies in $\mathrm{CsPbX}_{3}$ PNCs using Lewis base ligands can be predicted by the hard-soft acidsbases theory ${ }^{32}$ : harder species such as alkylcarboxylates and carbonates are ineffective due to a hard-soft mismatch between hard groups and soft $\mathrm{Pb}$ binding sites, whereas softer species such as alkylphosphonates and sulfonates are effective due to their higher binding affinity (Fig. 2b). Hydrogen bonding between the passivation agents and the organic cations of the perovskite structure has a critical role in determining the passivation effectiveness ${ }^{6}$ : diamine-based passivation agents, whose amino groups form weaker hydrogen bonding with organic cations, are more effective in coordinating with unsaturated $\mathrm{Pb}$ sites (Fig. 2c). Moreover, molecules with $\mathrm{P}=\mathrm{O}$ and $\mathrm{As}=\mathrm{O}$ end groups have shown strong binding energies with unsaturated $\mathrm{Pb}$ sites in reduced-dimensional perovskites, leading to high PLQYs ${ }^{34}$. An in-depth understanding of such structure-passivation relationships would be useful for rational design of optimal passivation agents. 
We note that PNCs often require additional considerations for the passivation agents although they share similar defect passivation mechanisms with bulk perovskites. For example, the commonly used surface ligands (such as oleic acid and oleylamine) are highly labile and easily desorb from the surface during the purification, leading to a high density of dangling bonds at the surface of $\mathrm{PNCs}^{35}$. In addition, the residual long-chain organic ligands usually deteriorate the charge-transport properties of the resulting emissive layers. Therefore, alternative passivation ligands with stronger binding affinity to PNCs and less negative effects on the electrical properties of the emissive layers (for instance, phosphonates, zwitterionic salts, and metal halides, see Fig. 2b) are highly desired and have been explored ${ }^{13}$. These useful strategies have significantly improved the optoelectronic properties and stability of PNCs, benefiting the resulting PeLEDs ( ref $^{13,36}$ ).

Controlling nonradiative Auger recombination. Auger recombination is a nonradiative process in which an electron-hole pair passes recombination energy to another charge carrier. In the case of a high excitation density where Auger recombination is dominant with $C N^{2}>>$ $(B N+A)$ in Equation (3) or $k_{2} N>>\left(k+k_{\text {trap }}\right)$ in Equation (5), the PLQY is inversely related to the excitation density (Fig. 1b, c and e). It has been reported that Auger recombination can result in significant efficiency roll-off at high current densities in LEDs ${ }^{37}$.

In $3 \mathrm{D}$ bulk perovskites, of which the $C$ value is around $10^{-28} \sim 10^{-29} \mathrm{~cm}^{6} \mathrm{~s}^{-1}$ (refs. ${ }^{18,38}$ ), Auger recombination is usually dominant at excitation densities higher than $10^{17} \mathrm{~cm}^{-3}$ (Fig. $1 \mathrm{~b}, \mathrm{c})$, indicating that 3D bulk perovskites are promising for high-brightness LEDs and laser diodes. In quantum-confined quasi-2D perovskites, Auger recombination becomes dominant at a lower charge carrier density (Fig. 1e) and could be one or two orders of magnitude faster ${ }^{17}$. A promising mitigation strategy in PeLEDs based on mixed dimensional perovskites is to introduce a higher number of phases with large $n$ values to reduce local carrier density, ensuring high EQEs at high current densities ${ }^{39}$. 
Although Auger recombination in NCs usually increases remarkably with reducing crystal sizes, the situation is different in PNCs, where Auger recombination shows a much weaker volume dependence compared to conventional QDs, deviating from the common volume scaling $(1 / \text { volume })^{40,41}$. This phenomenon is more pronounced in the weak confinement region, resulting in very small difference of Auger recombination rates of PNCs compared with bulk perovskites ${ }^{42}$. Auger recombination was demonstrated to be slower in organic cation-containing PNCs compared to all-inorganic ones ${ }^{42}$. These observations suggest that PNCs may outperform conventional QDs under high excitation densities, and they call for further understanding of the mechanisms determining Auger losses in perovskites.

\section{Device engineering and characterizations of PeLEDs}

Highly emissive perovskite layers are only one ingredient to realize high-efficiency PeLEDs; other key elements include balanced and efficient charge injection as well as high light outcoupling efficiency. Typically, PeLEDs have a device structure in which the perovskite emissive layer is sandwiched between electron- and hole-transport interlayers. The device structure is similar to those of solution-processed organic LEDs (OLEDs) and conventional QD-based LEDs (QLEDs), from which we can readily borrow rich experience, including the selection of interfacial materials, engineering of energy level alignments at the interfaces, manipulation of charge injection, and design of light outcoupling techniques.

Despite these similarities, two features are unique to PeLEDs: strong physical/chemical interactions between perovskites and interlayers; and ion migration. These two features have impacts on both the device optimization and reliable characterization of the device performance. In addition, perovskites also provide opportunities for facile control of light outcoupling, which will also be discussed in this section.

Interface engineering of PeLEDs. The physical properties of interlayers can affect the crystallinities, morphologies, defect properties and energy level structures of perovskite layers 
deposited on top of them ${ }^{43}$. For example, studies on solar cells have revealed that the surface wetting properties of bottom interlayers affect the crystallization process of the subsequently deposited perovskite films ${ }^{44}$. In addition, different surface properties of the perovskite layers can also affect the stacking of organic molecules on top of them, resulting in significant morphological and electrical changes of the charge-transport contacts ${ }^{45}$.

Chemical interactions at the perovskite-interlayer interfaces and their impacts on the performance of PeLEDs are more complicated and deserve further investigations. Functional passivating moieties (such as Lewis base/acid groups), if grafted on interfacial materials, are likely to induce additional healing of surface defects ${ }^{46}$. Beyond passivation, other chemical reactions can occur between perovskite precursors and interlayers; for instance, the alkaline nature of the $\mathrm{ZnO}$ interlayer induces efficient deprotonation of the organic cations and promotes perovskite crystallization ${ }^{47}$. As such, rational control of the precursor stoichiometry has enabled high-quality $\mathrm{FA}_{x} \mathrm{Cs}_{1-x} \mathrm{PbI}_{3}$ perovskite films on a $\mathrm{ZnO}$ interlayer, while the same precursor failed to produce perovskite films on $\mathrm{TiO}_{x}$ and $\mathrm{SnO}_{2}$ interlayers (Fig. 3a) ${ }^{47}$.

In addition to the impacts on device engineering, these strong physical/chemical interactions between perovskites and interlayers also suggest that special attention should be paid to the characterizations of perovskite films, especially the in-situ crystallized polycrystalline films on different substrates. For instance, single carrier devices, which are commonly used to evaluate charge injection/transport balance in OLEDs and QLEDs, might not be reliable in PeLEDs due to the possible changes of the perovskite active layers deposited on different charge-transport interlayers.

For the emissive layers assembled from PNCs, which are more sensitive to most of the commonly used organic solvents compared to conventional colloidal QDs, vacuum deposition of the subsequent charge-transport layers is often employed to avoid damage of the emissive layers $^{48}$. For successful solution processing of charge-transport layers on top, rationally 
designed surface ligands or crosslinking of the PNCs are necessary to improve the solvent resistance of these emissive layers to organic solvents ${ }^{49,50}$.

Light outcoupling in PeLEDs. Perovskite emissive layers generally possess a high refractive index, which potentially limits the outcoupling efficiency of PeLEDs as predicted by the rayoptics theory. However, detailed optical simulations indicate higher outcoupling efficiency in PeLEDs, leading to maximum EQEs of $25 \%$ and $20 \%$ for PeLEDs based on 3D perovskites and mixed dimensional perovskites, respectively, under the assumption of planar structured films without any light extraction technologies ${ }^{51}$. To further improve the external efficiencies, the same light extraction technologies developed in OLEDs and QLEDs (for instance, the use of a nanophotonic substrate ${ }^{52}$ or nanoimprinted moth-eye nanostructures combined with a halfball lens ${ }^{53}$ ) have been proven useful in enhancing light outcoupling and peak EQEs of PeLEDs as well. In addition, for PeLEDs with strong self-absorbing perovskite emissive layers, various filtering structures and reduced electrode area are expected to show a greatly enhanced light outcoupling efficiency, owing to the benefits of high photon recycling combined with a reduction in the amount of photons trapped in the substrate and waveguide modes ${ }^{54}$.

A further strategy to improve light outcoupling relies on modulating the morphology of perovskite films. Perovskite emissive layers consisting of isolated submicrometre-sized platelets (Fig. 3b) exhibit a much-enhanced light outcoupling efficiency approaching 30\%, leading to NIR PeLEDs with a high peak EQE of $20.7 \%$ (ref. ${ }^{8}$ ). The simple light extraction strategy through morphology control of the perovskite emissive layers retains the wavelengthand viewing-angle-independent electroluminescence (EL) and avoids the use of complicated manufacturing processes ${ }^{8}$. We note that, in contrast to the early-stage film optimization strategies which emphasize the film uniformness, discontinuous film morphology does not necessarily result in electrical issues, as long as the leakage current and electrical shunts through the gaps of the isolated perovskite grains can be properly suppressed ${ }^{6,8,55}$. 
Another approach for achieving high light outcoupling efficiency is to use light emitters with horizontally oriented (in-plane) transition dipole moments, a strategy that is widely used in OLEDs and yet to be explored in PeLEDs. Theoretical simulations predict a maximum light outcoupling efficiency of around 46\% for OLEDs based on light emitters with both PLQYs and orientation factor approaching unity ${ }^{56}$. Encouragingly, anisotropic photon emission has been observed in $\mathrm{PNCs}^{57}$, suggesting that high light outcoupling efficiencies in PeLEDs are possible by using perovskites with in-plane transition dipole moments.

Device characterizations of PeLEDs. The characterization methods for PeLEDs follow the standards established in other LED technologies ${ }^{58}$. However, the well-acknowledged ion migration in perovskites brings challenges in reliably determining the parameters and this calls for further attention. In particular, the performance parameters of PeLEDs, including current densities, EQEs, luminance/radiance and efficiency roll-off, often depend on how the measurements are performed. For example, a hysteresis in EQE-voltage curves under different scanning directions was reported ${ }^{5}$. In addition, EQE enhancement by repeated voltage scans was observed, ascribed to defect filling caused by ion migration under electrical stress ${ }^{59}$. It has also been demonstrated that the peak values and shapes of the EQE curves are dependent on the scan speed during the measurements ${ }^{21}$.

As an emerging research field that is attracting increasing attention, reliable measurements of the device parameters are critical for the development of PeLEDs. We suggest providing details of the measurements, such as scan step, delay time, scan speed and the use of pre-bias stress protocols. In addition to the commonly used EQE- and brightness-current density curves (Fig. 3c), we propose to provide stabilized EQEs and brightness at a constant current density to minimize the effect of scanning methods on the device performance (Fig. 3d). Although the values measured under a constant current density could be slightly different from those measured with scanning current densities, the former represents the values under practical 
operating conditions of LEDs. Note that measuring stabilized power output has been widely adopted in the perovskite photovoltaic community to accurately determine the performance ${ }^{60}$. In addition, for spectrally unstable devices, it is necessary to provide both the EL spectra and EQEs at different brightness or at different driving voltages/current densities.

Device operational stability is another critical parameter of PeLEDs. In the LED luminaire industry, operational stability (lifetime) is typically recognized as the operating time until lumen maintenance has diminished to a certain level. Similar to this industrial standard, in literature, the operational stability of a visible LED is often evaluated based on the time required for the luminance to drop to a certain level (for instance, $50 \%$; that is, $T_{50}$ ) of its initial value under continuous operating conditions ${ }^{7,9,28,61}$. As shown in Fig. 3e, slow increase of the luminance ranging from minutes to hours $\left(T_{\text {ramp }}\right)$ is often observed in stability measurements of PeLEDs ${ }^{7,28,43,46,62}$. In this case, the time needed to drop to $50 \%$ of the peak luminance $\left(T_{\text {decay }}\right)$ would be a more reasonable value to evaluate the device's operational stability (Fig. 3e), as there is no evidence showing that the ramp process is part of the degradation. In addition, the operational stability of PeLEDs strongly depends on the environmental temperature (Fig. 3f $)^{63}$, which should also be clearly stated along with the device stability results.

For devices with relatively poor stability, which is the case for most PeLEDs demonstrated at this stage, we would suggest directly measuring the $T_{50}$. For highly stable PeLEDs, accelerated tests of the operational stability at a high luminance can be used to predict the $T_{50}$ at a lower luminance (for instance, $100 \mathrm{~cd} \mathrm{~m}^{-2}$ ). The prediction is based on an empirical scaling law described in Equation (6):

$T_{50} \times L_{0}^{a}=$ Constant

where $a$ is the acceleration factor fitted based on the measured lifetimes at different initial luminance $\left(L_{0}\right)$ values $^{64}$. We emphasize that the $a$ depends on the materials and the device architectures. As a result, for highly stable PeLEDs where we need to use Equation (6), it is 
necessary to determine the $a$ value based on sufficient data sets collected from stability tests under different initial luminance values. We also note that the degradation mechanisms of PeLEDs might be different from the other LEDs, and hence systematical studies and in-depth understanding of the degradation mechanisms are required. For example, ion migration could screen external electric fields, causing changes in diode characteristics. In addition, the ions tend to localize at the interfaces or grain boundaries, leading to the changes of interfacial properties and formation of defect sites.

For non-visible PeLEDs (for instance, NIR ones), where luminance is not relevant, we propose to measure the radiance decay instead when reporting their operational stability. This would allow the community to reasonably compare the device lifetimes of non-visible PeLEDs at a certain level of light output, as already done for visible PeLEDs.

\section{Key challenges ahead in PeLEDs}

Rapid progress of PeLEDs has been made in the past several years, yet there are critical challenges to be tackled to foster further advances of this promising technology. In this section, we identify key challenges and discuss possible strategies to address them: improving the performance of red and blue PeLEDs, enhancing the stability of PeLEDs, and solving toxicity issue of lead.

High-performance red and blue PeLEDs. Despite the great success in high-performance green and NIR PeLEDs, it is still challenging to achieve high-efficiency red and blue devices with high luminance (Fig. 4a), especially those with the CIE coordinates approaching the Rec. 2020 specified red $(0.708,0.292)$ and blue $(0.131,0.046)$ primary colors that are desired for ultra-high-definition displays.

A straightforward strategy to approach these desired red and blue colors is to use perovskite emissive layers with well-adjusted mixed halide compositions: mixed chloride-bromide for blue and mixed bromide-iodide for red emission. Although continuous bandgap tuning via 
varying halide ions is one of the most attractive properties of perovskite emitters, practical applications of this strategy are limited by color instability of the resulting PeLEDs ${ }^{9,65}$. For instance, in the case of red PeLEDs based on mixed bromide-iodide perovskite emissive layers, $\mathrm{Br}$ and I ions tend to segregate into $\mathrm{Br}$ - and I-rich domains under the electric field, resulting in shifts of the EL spectra and changes of emission colors during device operation (Fig. 4b) ${ }^{66}$; a similar phenomenon was also observed in blue PeLEDs employing mixed chloride-bromide perovskite emissive layers ${ }^{49}$.

Although they are challenging, we believe that it is too early to rule out the possibility of using mixed halide perovskites for color-stable and efficient red/blue emission. Experimental and theoretical calculations suggest that phase segregation is mediated by ionic defects, especially by vacancies ${ }^{67}$. Therefore, particular attention should be paid to defect passivation to eliminate ion migration pathways. In addition, previous investigations on perovskite photovoltaics suggested that photo-induced phase segregation is thermodynamic in nature; hence it can be driven by free-energy differences linked to composition or electronic structure ${ }^{68}$. Local strains induced by lattice mismatch may also facilitate ion migration ${ }^{69}$. Therefore, a high compositional homogeneity in mixed halide perovskite films with low free energy and low local strain could be favorable for suppressing phase segregation, although it is a challenging task owing to widely observed non-equilibrium crystal growth in these materials ${ }^{70}$. It has also been proposed that light-induced phase-separation in mixed halide perovskites is negligible when charge carrier density is below a certain threshold ${ }^{71}$. This might explain previous results showing improved spectral stability for PeLEDs based on mixed halide perovskites operated at low current densities ${ }^{9,62}$. These findings indicate that achieving color-stable PeLEDs with mixed halide compositions might be feasible, and call for further understanding of the ion segregation mechanisms, especially under the electric field. 
An alternative strategy to approach the Rec. 2020 primary blue (red) colors is to introduce quantum confinement effects in pure bromide (iodide)-based perovskites. The enhanced quantum confinement can be achieved through either using mixed dimensional perovskites or synthesizing perovskite QDs. However, considerable challenges also accompany these quantum-confined structures.

In mixed dimensional perovskites, emission mostly occurs from the phase with the smallest bandgap, owing to efficient energy transfer from the large bandgap phases ${ }^{26,27}$. Because there is usually a wide dimensional distribution, a high amount of large-sized organic cations is required to push the emission towards the desired blue and red colors ${ }^{61,72}$; however, poor charge-transport properties, multiphase-induced peak broadening and even electroluminescence peak shifts are widely observed in these emissive layers ${ }^{72,73}$. In addition, this can also bring about more $n=1$ phases which are usually less efficient emitters due to the strong electron-phonon coupling. Therefore, precise dimensional control towards narrow dimensional distribution, that would reduce the need for large organic cations and improve charge transport, is highly desired to realize Rec. 2020 primary blue and red emission in mixed dimensional perovskites (Fig. 4c $)^{74}$. Crystallization control approaches, such as using antisolvent techniques or carefully screening large cations and additives, would be promising to decrease the dimensional distribution ${ }^{74,75}$. In addition to modulating dimensional distribution, controlling the vertical orientation of the perovskite phases, favorable for charge transport in PeLEDs, might also help to improve device performance ${ }^{76}$.

For QDs, the quantum confinement is typically obtained when the crystal size of a material is comparable to or smaller than its exciton Bohr diameter. The exciton Bohr diameters for $\mathrm{CsPbBr}_{3}$ and $\mathrm{CsPbI}_{3}$ are $7 \mathrm{~nm}$ and $12 \mathrm{~nm}$, respectively ${ }^{15}$; the values are even smaller for their organic-inorganic hybrid counterparts ${ }^{77}$. This imposes strict limits to the crystal size, making it challenging to synthesize such small QDs with desired blue/red emission and good stability. 
Poor size uniformity might be another challenge for these small QDs, inducing spectral broadening and leading to poor color purity ${ }^{13}$. To mitigate these limits of QDs, shape engineering, such as the use of colloidal nanoplatelets, also called colloidal quantum wells (CQWs), could be a useful approach ${ }^{78}$. As the quantum confinement in these systems is hardly affected by the lateral size distribution, the size requirement is only on the thickness of the CQWs and hence less stringent, beneficial for achieving narrow emission linewidth. For example, a sharp photoluminescence spectrum with the FWHM of $18 \mathrm{~nm}$ has been realized in blue emissive $\mathrm{CsPbBr}_{3} \mathrm{CQWs}$ with well controlled thickness (Fig. 4d, left panel) ${ }^{79}$. These CQWs can also form large nanocrystals through self-assembly (Fig. 4d, right panel) while preserving narrow emission from the CQWs, which is promising for PeLEDs ${ }^{80,81}$.

Successful fabrication of high-performance PeLEDs with blue/red emission satisfying the display purpose might require a combination of strategies leveraging on mixed halides, the quantum confinement effect, and other materials composition engineering (such as A- or B-site alloying or doping). For instance, the introduction of $\mathrm{Rb}^{+}$into $\mathrm{CsPbBr} 3$ increases the bandgap owing to the tilting of the $\left[\mathrm{PbBr}_{6}\right]^{4-}$ octahedra and the reduced overall orbital overlap ${ }^{82}$. It is therefore attractive to shift the emission towards the blue by incorporating $\mathrm{Rb}^{+}$, which is also compatible with the other strategies for bandgap tuning. In fact, blue emission within the range of about 450-490 $\mathrm{nm}$ has been demonstrated in $\mathrm{PEA}_{2}\left(\mathrm{Rb}_{x} \mathrm{Cs}_{1-x}\right)_{2} \mathrm{~Pb}_{3} \mathrm{Br}_{10}$ mixed-dimensional perovskites, where PEA is phenethylammonium (Fig. 4e $)^{83}$. Introducing impurity metal ions into the perovskite lattice provides an additional synthetic tool to tune the bandgaps of the PNCs. For instance, zinc- or cadmium-doped $\mathrm{CsPbBr}_{3}$ PNCs present considerably blue-shifted emission owing to lattice contraction (Fig. 4f) ${ }^{84}$. This approach could be difficult to achieve in polycrystalline films formed in-situ, because of the high formation energies of the doped perovskites, in which the impurity metal ions tend to locate on grain surfaces. 
Stability of PeLEDs. As a young technology, the operational stability of PeLEDs remains a big challenge limiting their practical applications. Currently, the most stable PeLEDs are recorded with NIR devices ${ }^{8}$. Nevertheless, visible PeLEDs show $T_{50}$ of hundreds of hours at most at a low luminance of $100 \mathrm{~cd} \mathrm{~m}^{-2}$ (ref. ${ }^{85}$ ), far behind those of OLEDs and QLEDs. Most of the blue PeLEDs reported so far can only last for tens of seconds to minutes ${ }^{61,83}$. Inspired by the development of perovskite photovoltaics ${ }^{86}$, we consider that the factors responsible for the poor stability of PeLEDs could mainly include ion migration, thermal instability, and interfacial instability.

Besides the impact on phase stability of mixed halide perovskites discussed above, ion migration has also been regarded as a critical issue affecting the long-term stability of perovskite optoelectronic devices ${ }^{67}$. Ion migration is generally associated with defect formation, interfacial charge and ion accumulation, degradation of interlayers and corrosion of electrodes ${ }^{67}$. It is generally believed that point defects, especially halide vacancies, serve as ion migration channels ${ }^{87}$. Therefore, reducing defect density with rational passivation and efficient crystallization control would be effective ways to mitigate ion migration ${ }^{6,55}$. Fortunately, PeLEDs are more tolerant to additives than perovskite photovoltaics, owing to less stringent requirements on charge-carrier mobility. Hence, various additives are allowed in perovskite films at a relatively high content, making it possible to heal the defects or immobilize the mobile ions by a variety of chemical bonding or physical interactions. In addition to the long-term stability of PeLEDs, ion migration might also affect the response time of the devices during practical display operations. Encouragingly, similar dynamic behaviours as those observed in OLEDs were demonstrated by transient EL investigations of PeLEDs ${ }^{88}$, and the operation frequency of PeLEDs can reach tens of megahertz ${ }^{11}$.

Thermal instability of perovskite emitters is not only related to materials decomposition, but also to the change of dimensional distribution upon heating. The latter is particularly serious 
in some mixed dimensional perovskite films, which usually undergo a dimensional transition toward large- $n$ phases upon heating ${ }^{89}$. In addition, we notice that most of the highly emissive perovskite films are prepared with a short-term annealing procedure at a low temperature $(<100$ ${ }^{\circ} \mathrm{C}$ for several minutes $)^{61,90}$. Further increasing the annealing temperature or duration usually leads to PLQY dropping ${ }^{90}$. Therefore, Joule heating during device operation might cause permanent degradation of the perovskite emissive layers.

In addition to the issues caused by mobile ions and thermal instability of perovskites, the selection of electrodes and charge-transport layers can also significantly affect the device operational stability ${ }^{55,63,91}$. Previous investigations have revealed that drift and diffusion of metal ions from the electrodes into perovskites can result in device degradation due to redox reactions (for instance, between $\mathrm{Al}^{0}$ and $\mathrm{Pb}^{2+}$ ) 91 . This might be more serious in PeLEDs due to the electric field-induced metal ion diffusion. In addition, it was demonstrated that the EL decay could be much quicker than the PL decay in PeLEDs, indicating the degradation of the interfaces between the perovskite emissive layer and charge-transport layers ${ }^{55,63}$. Ideal chargetransport layers should have excellent interfacial and morphological stability upon heating and could also protect the perovskite layers from metal ions in the electrodes. Currently, the device structures and interlayer materials for PeLEDs follow those established in OLEDs. However, perovskites are much more sensitive to interfacial chemical and physical environment ${ }^{47}$, and hence more targeted designs are necessary to improve the device lifetime.

Lead-free perovskites. The presence of lead in perovskite optoelectronic devices raises significant concerns about their toxicity, even though the lead content in perovskite optoelectronic devices could meet the current criteria for commercial products as previously evaluated in perovskite photovoltaics ${ }^{92}$. One critical reason is that lead halide perovskites have high solubility in water, making them readily leach into the environment in the case of encapsulant failure ${ }^{92}$. Before a clear consensus on the toxicity issue is reached, increasing 
efforts should be made in parallel to search for alternative compounds which are environmentally friendly and equally performing.

During the past several years, a range of promising lead-free materials have been developed, including $3 \mathrm{D} \mathrm{ABX}_{3}$ perovskites (mainly based on $\mathrm{Sn}^{2+}$ ), vacancy ordered perovskites (such as $\mathrm{Cs}_{2} \mathrm{SnI}_{6}$ ), halide double perovskites (such as $\mathrm{Cs}_{2} \mathrm{AgInCl}_{6}, \mathrm{Cs}_{2} \mathrm{AgBiBr}_{6}$ ), and non-perovskite metal halides with various metal ions and dimensionalities ${ }^{93}$. These lead-free perovskites and perovskite derivatives, some of which have shown comparable PLQYs to lead halide perovskites, represent a rich library for LED applications. When attempted in LEDs, these materials (such as tin-based perovskites ${ }^{94}, \mathrm{Cs}_{2} \mathrm{AgInCl}_{6}$ derivatives ${ }^{12}, \mathrm{~A}_{3} \mathrm{~B}_{2} \mathrm{X}_{9}$-type materials ${ }^{95}$ and copper halides ${ }^{96}$ ) show relatively low efficiencies for now. Although the design rules to target promising materials from such a big library are currently lacking, these lead-free compounds might have better chances for LEDs than photovoltaics, considering larger tolerance on exciton binding energies, bandgaps, and charge carrier mobilities of the materials in LEDs. A combination of experimental and theoretical investigations, maybe involving machine learning, can hopefully help to search for the right lead-free materials for highperformance $\operatorname{LEDs}^{97}$.

\section{Conclusions and outlook}

Recent advances of PeLEDs indicate their bright future in the areas of displays, lighting and optical communications. Their properties potentially go beyond these areas; for example, excellent performance of PeLEDs and hybrid LEDs based on inorganic QDs and perovskites ${ }^{98,99}$ in NIR region may bring about new applications in biomedical diagnosis and a variety of wearable electronic devices. Here we discuss the outlook for further development of white PeLEDs and the potential applications of perovskite emitters in printable and flexible electronics as well as electrically pumped lasers, aiming to shed light on these promising future directions. 
White PeLEDs. In addition to the three primary colors (blue, green and red), perovskites also offer great opportunities for direct white light emission. White light can be generated by using a single white-emitting material or a set of luminophores of distinct colors. Luminophores are assembled in several ways, such as single- or multi-emissive-layer white LEDs or hybrid LEDs combining color-down-conversion emitters with blue/ultraviolet chips. Although efficient hybrid white LEDs combining perovskite emitters with commercial inorganic LED chips have been demonstrated, there are few examples of white PeLEDs that show white electroluminescence directly from the perovskite emitters ${ }^{12,100}$. One promising strategy is to take advantage of the emission from self-trapped excitons (STEs). In the simplified mechanism for STE emission (Fig. 5a), free excitons undergo fast relaxation to various self-trapped states with different energies lying within the bandgap, yielding broadband emission that is desired for indoor lighting. STEs have been widely observed in 2D perovskites, lead free perovskites and other metal halides ${ }^{101}$. For instance, white light emission from STEs was achieved in doped double perovskites (Fig. 5b) ${ }^{12}$. However, the performance of these white PeLEDs needs further improvement, and more efforts are required to exploit the potential of perovskite materials for white light generation.

Printable and flexible electronics. Perovskite emitters are compatible with low-temperature solution-based manufacturing techniques, such as inkjet printing, roll-to-roll and 3D printing, providing great potential in scale-up production and flexible electronics. For example, multicolour pixel arrays based on 3D-printed perovskite nanowires have been demonstrated, where the emission colour can be controlled by leveraging anisotropy property of the emitters with polarizing filters ${ }^{102}$. High-performance flexible PeLEDs fabricated on various light-weight substrates, such as carbon nanotubes and silver nanowires, have also been demonstrated ${ }^{103,104}$. These devices showed excellent mechanical robustness with negligible performance loss after a bending test of up to 10,000 cycles $^{104}$. Pixelated touch-responsive PeLEDs for instantaneous 
visualization of pressure mapping further expand the potential of this technology to other applications including robotics, motion detection and fingerprint identification ${ }^{105}$. For highresolution devices with enhanced performance, further improvements in ink design, device engineering and associated printing and encapsulation technologies are still necessary.

Electrically pumped perovskite lasers. Solution-processed electrically pumped laser diodes have been intensively studied since 1990s but are hard to achieve using organic emitters and conventional QDs ${ }^{106}$. Perovskites, which show low trap density $\left(<10^{16} \mathrm{~cm}^{-3}\right)$, high chargecarrier mobility $\left(>10 \mathrm{~cm}^{2} \mathrm{~V}^{-1} \mathrm{~s}^{-1}\right)$, and low Auger recombination coefficient $\left(10^{-28}\right.$ to $10^{-29} \mathrm{~cm}^{6}$ $\left.\mathrm{s}^{-1}\right)^{2}$, may offer greater opportunities towards this ambitious goal. So far, a range of optically pumped perovskite lasers with tunable emission (from 390 to $800 \mathrm{~nm}$ ), ultra-low threshold (0.2$40 \mu \mathrm{J} \mathrm{cm}^{-2}$ ), and high optical gain (as high as 3,200 $\mathrm{cm}^{-1}$ for $\mathrm{CsPbBr}_{3}$ PNCs) have been demonstrated ${ }^{24}$. The achievement of lasing usually requires a high threshold carrier density of around $10^{18} \mathrm{~cm}^{-3}$, corresponding to a roughly estimated threshold current density of at least hundreds of $\mathrm{A} \mathrm{cm}^{-2}$ under electrical excitation ${ }^{107}$. Encouragingly, short-pulse driven PeLEDs have shown a negligible Auger loss although they were operated at high current densities exceeding $150 \mathrm{~A} \mathrm{~cm}^{-2}$; in this case, their efficiency roll-off is dominated by Joule heating and charge imbalance ${ }^{108}$. It has also been demonstrated that the excitation threshold dramatically increases with rising temperature (Fig. 5c) ${ }^{109}$. Rational designs are thus desired to develop perovskite materials with temperature insensitive thresholds and devices with thermal dissipation, respectively. Along this line, thermal management in devices have successfully resulted in PeLEDs with ultra-high radiance at around $2 \mathrm{kA} \mathrm{cm}^{-2}$ (Fig. 5d ${ }^{110}$.

To conclude, we have discussed the fundamental requirements for achieving highperformance PeLEDs from both material and device perspectives, and highlighted the key challenges towards high-performance blue and red PeLEDs, long-term device stability and lead-free PeLEDs. These challenges require an interdisciplinary approach for further 
breakthroughs to create industrial paths. Combining the considerable advances already made by the community and the outstanding properties of perovskites, we believe that PeLEDs offer unique advantages for future applications.

\section{References}

1. Stranks, S. D. \& Snaith, H. J. Metal-halide perovskites for photovoltaic and light-emitting devices. Nat. Nanotechnol. 10, 391-402 (2015).

2. Quan, L. N. et al. Perovskites for next-generation optical sources. Chem. Rev. 119, 74447477 (2019).

3. Era, M., Morimoto, S., Tsutsui, T. \& Saito, S. Organic-inorganic heterostructure electroluminescent device using a layered perovskite semiconductor $\left(\mathrm{C}_{6} \mathrm{H}_{5} \mathrm{C}_{2} \mathrm{H}_{4} \mathrm{NH}_{3}\right)_{2} \mathrm{PbI}_{4}$. Appl. Phys. Lett. 65, 676-678 (1998).

4. Chondroudis, K. \& Mitzi, D. B. Electroluminescence from an organic-inorganic perovskite incorporating a quaterthiophene dye within lead halide perovskite layers. Chem. Mater. 11, 3028-3030 (1999).

5. Tan, Z.-K. et al. Bright light-emitting diodes based on organometal halide perovskite. Nat. Nanotechnol. 9, 687-692 (2014).

6. Xu, W. et al. Rational molecular passivation for high-performance perovskite lightemitting diodes. Nat. Photon. 13, 418-424 (2019).

7. Lin, K. et al. Perovskite light-emitting diodes with external quantum efficiency exceeding 20 per cent. Nature 562, 245-248 (2018).

8. Cao, Y. et al. Perovskite light-emitting diodes based on spontaneously formed submicrometre-scale structures. Nature 562, 249-253 (2018). 
9. Chiba, T. et al. Anion-exchange red perovskite quantum dots with ammonium iodine salts for highly efficient light-emitting devices. Nat. Photon. 12, 681-687 (2018).

10. Zhao, B. et al. High-efficiency perovskite-polymer bulk heterostructure light-emitting diodes. Nat. Photon. 12, 783-789 (2018).

11. Bao, C. et al. Bidirectional optical signal transmission between two identical devices using perovskite diodes. Nat. Electron. 3, 156-164 (2020).

12. Luo, J. et al. Efficient and stable emission of warm-white light from lead-free halide double perovskites. Nature 563, 541-545 (2018).

13. Shamsi, J., Urban, A. S., Imran, M., De Trizio, L. \& Manna, L. Metal halide perovskite nanocrystals: synthesis, post-synthesis modifications, and their optical properties. Chem. Rev. 119, 3296-3348 (2019).

14. Huang, H., Bodnarchuk, M. I., Kershaw, S. V., Kovalenko, M. V. \& Rogach, A. L. Lead halide perovskite nanocrystals in the research spotlight: stability and defect tolerance. $A C S$ Energy Lett. 2, 2071-2083 (2017).

15. Protesescu, L. et al. Nanocrystals of cesium lead halide perovskites $\left(\mathrm{CsPbX}_{3}, \mathrm{X}=\mathrm{Cl}, \mathrm{Br}\right.$, and I): novel optoelectronic materials showing bright emission with wide color gamut. Nano Lett. 15, 3692-3696 (2015).

16. Yu, D., Cao, F., Gao, Y., Xiong, Y. \& Zeng, H. Room-temperature ion-exchangemediated self-assembly toward formamidinium perovskite nanoplates with finely tunable, ultrapure green emissions for achieving Rec. 2020 displays. Adv. Funct. Mater. 28, 1800248 (2018). 
17. Xing, G. et al. Transcending the slow bimolecular recombination in lead-halide perovskites for electroluminescence. Nat. Commun. 8, 14558 (2017).

18. Herz, L. M. Charge-carrier dynamics in organic-inorganic metal halide perovskites. Annu. Rev. Phys. Chem. 67, 65-89 (2016).

19. Manser, J. S. \& Kamat, P. V. Band filling with free charge carriers in organometal halide perovskites. Nat. Photon. 8, 737-743 (2014).

20. Cho, H. et al. Overcoming the electroluminescence efficiency limitations of perovskite light-emitting diodes. Science 350, 1222-1225 (2015).

21. Xiao, Z. et al. Efficient perovskite light-emitting diodes featuring nanometre-sized crystallites. Nat. Photon. 11, 108-115 (2017).

22. Li, G. et al. Efficient light-emitting diodes based on nanocrystalline perovskite in a dielectric polymer matrix. Nano Lett. 15, 2640-2644 (2015).

23. Feldmann, S. et al. Photodoping through local charge carrier accumulation in alloyed hybrid perovskites for highly efficient luminescence. Nat. Photon. 14, 123-128 (2020).

24. Wei, Q. et al. Recent progress in metal halide perovskite micro- and nanolasers. Adv. Opt. Mater. 7, 1900080 (2019).

25. Dutta, A., Behera, R. K., Pal, P., Baitalik, S. \& Pradhan, N. Near-unity photoluminescence quantum efficiency for all $\mathrm{CsPbX}_{3}(\mathrm{X}=\mathrm{Cl}, \mathrm{Br}$, and I) perovskite nanocrystals: a generic synthesis approach. Angew. Chem. 131, 5608-5612 (2019).

26. Wang, N. et al. Perovskite light-emitting diodes based on solution-processed selforganized multiple quantum wells. Nat. Photon. 10, 699-704 (2016). 
27. Yuan, M. et al. Perovskite energy funnels for efficient light-emitting diodes. Nat. Nanotechnol. 11, 872-877 (2016).

28. Qin, C. et al. Triplet management for efficient perovskite light-emitting diodes. Nat. Photon. 14, 70-75 (2020).

29. Gao, Y. et al. Molecular engineering of organic-inorganic hybrid perovskites quantum wells. Nat. Chem. 11, 1151-1157 (2019).

30. Chen, B., Rudd, P. N., Yang, S., Yuan, Y. \& Huang, J. Imperfections and their passivation in halide perovskite solar cells. Chem. Soc. Rev. 48, 3842-3867 (2019).

31. Ball, J. M. \& Petrozza, A. Defects in perovskite-halides and their effects in solar cells. Nat. Energy 1, 16149 (2016).

32. Nenon, D. P. et al. Design principles for trap-free $\mathrm{CsPbX}_{3}$ nanocrystals: enumerating and eliminating surface halide vacancies with softer Lewis bases. J. Am. Chem. Soc. 140, 17760-17772 (2018).

33. Lee, S. et al. Versatile defect passivation methods for metal halide perovskite materials and their application to light-emitting devices. Adv. Mater. 31, 1805244 (2019).

34. Quan, L. N. et al. Edge stabilization in reduced-dimensional perovskites. Nat. Commun. 11, 170 (2020).

35. De Roo, J. et al. Highly dynamic ligand binding and light absorption coefficient of cesium lead bromide perovskite nanocrystals. ACS Nano 10, 2071-2081 (2016).

36. Chiba, T. \& Kido, J. Lead halide perovskite quantum dots for light-emitting devices. $J$. Mater. Chem. C 6, 11868-11877 (2018). 
37. Bae, W. K. et al. Controlling the influence of Auger recombination on the performance of quantum-dot light-emitting diodes. Nat. Commun. 4, 2661 (2013).

38. Wehrenfennig, C., Eperon, G. E., Johnston, M. B., Snaith, H. J. \& Herz, L. M. High charge carrier mobilities and lifetimes in organolead trihalide perovskites. Adv. Mater. 26, 15841589 (2014).

39. Zou, W. et al. Minimising efficiency roll-off in high-brightness perovskite light-emitting diodes. Nat. Commun. 9, 608 (2018).

40. Makarov, N. S. et al. Spectral and dynamical properties of single excitons, biexcitons, and trions in cesium-lead-halide perovskite quantum dots. Nano Lett. 16, 2349-2362 (2016).

41. Castañeda, J. A. et al. Efficient biexciton interaction in perovskite quantum dots under weak and strong confinement. ACS Nano 10, 8603-8609 (2016).

42. Eperon, G. E., Jedlicka, E. \& Ginger, D. S. Biexciton Auger recombination differs in hybrid and inorganic halide perovskite quantum dots. J. Phys. Chem. Lett. 9, 104-109 (2018).

43. Zhang, L. et al. Ultra-bright and highly efficient inorganic based perovskite light-emitting diodes. Nat. Commun. 8, 15640 (2017).

44. Bi, C. et al. Non-wetting surface-driven high-aspect-ratio crystalline grain growth for efficient hybrid perovskite solar cells. Nat. Commun. 6, 7747 (2015).

45. Jung, E. H. et al. Efficient, stable and scalable perovskite solar cells using poly(3hexylthiophene). Nature 567, 511-515 (2019). 
46. Yang, X. et al. Efficient green light-emitting diodes based on quasi-two-dimensional composition and phase engineered perovskite with surface passivation. Nat. Commun. 9, $570(2018)$.

47. Yuan, Z. et al. Unveiling the synergistic effect of precursor stoichiometry and interfacial reactions for perovskite light-emitting diodes. Nat. Commun. 10, 2818 (2019).

48. Song, J. et al. Quantum dot light-emitting diodes based on inorganic perovskite cesium lead halides $\left(\mathrm{CsPbX}_{3}\right) . A d v$. Mater. 27, 7162-7167 (2015).

49. Li, G. et al. Highly efficient perovskite nanocrystal light-emitting diodes enabled by a universal crosslinking method. Adv. Mater. 28, 3528-3534 (2016).

50. Shi, Z. et al. Strategy of solution-processed all-inorganic heterostructure for humidity/temperature-stable perovskite quantum dot light-emitting diodes. ACS Nano 12, $1462-1472$ (2018).

51. Shi, X.-B. et al. Optical energy losses in organic-inorganic hybrid perovskite lightemitting diodes. Adv. Opt. Mater. 6, 1800667 (2018).

52. Zhang, Q. et al. Efficient metal halide perovskite light-emitting diodes with significantly improved light extraction on nanophotonic substrates. Nat. Commun. 10, 727 (2019).

53. Shen, Y. et al. High-efficiency perovskite light-emitting diodes with synergetic outcoupling enhancement. Adv. Mater. 31, 1901517 (2019).

54. Cho, C. et al. The role of photon recycling in perovskite light-emitting diodes. Nat. Commun. 11, 611 (2020). 
55. Wang, H. et al. Perovskite-molecule composite thin films for efficient and stable lightemitting diodes. Nat. Commun. 11, 891 (2020).

56. Kim, K.-H. et al. Phosphorescent dye-based supramolecules for high-efficiency organic light-emitting diodes. Nat. Commun. 5, 4769 (2014).

57. Jurow, M. J. et al. Tunable anisotropic photon emission from self-organized $\mathrm{CsPbBr}_{3}$ perovskite nanocrystals. Nano Lett. 17, 4534-4540 (2017).

58. Anaya, M. et al. Best practices for measuring emerging light-emitting diode technologies. Nat. Photon. 13, 818-821 (2019).

59. Zhao, L. et al. Electrical stress influences the efficiency of $\mathrm{CH}_{3} \mathrm{NH}_{3} \mathrm{PbI}_{3}$ perovskite light emitting devices. Adv. Mater. 29, 1605317 (2017).

60. Snaith, H. J. et al. Anomalous hysteresis in perovskite solar cells. J. Phys. Chem. Lett. 5, $1511-1515$ (2014).

61. Liu, Y. et al. Efficient blue light-emitting diodes based on quantum-confined bromide perovskite nanostructures. Nat. Photon. 13, 760-764 (2019).

62. Wang, Q. et al. Efficient sky-blue perovskite light-emitting diodes via photoluminescence enhancement. Nat. Commun. 10, 5633 (2019).

63. Miao, Y. et al. Stable and bright formamidinium-based perovskite light-emitting diodes with high energy conversion efficiency. Nat. Commun. 10, 3624 (2019).

64. Féry, C., Racine, B., Vaufrey, D., Doyeux, H. \& Cinà, S. Physical mechanism responsible for the stretched exponential decay behavior of aging organic light-emitting diodes. Appl. Phys. Lett. 87, 213502 (2005). 
65. Li, C.-H. A., Zhou, Z., Vashishtha, P. \& Halpert, J. E. The future is blue (LEDs): why chemistry is the key to perovskite displays. Chem. Mater. 31, 6003-6032 (2019).

66. Vashishtha, P. \& Halpert, J. E. Field-driven ion migration and color instability in redemitting mixed halide perovskite nanocrystal light-emitting diodes. Chem. Mater. 29, 5965-5973 (2017).

67. Yuan, Y. \& Huang, J. Ion migration in organometal trihalide perovskite and its impact on photovoltaic efficiency and stability. Acc. Chem. Res. 49, 286-293 (2016).

68. Brivio, F., Caetano, C. \& Walsh, A. Thermodynamic origin of photoinstability in the $\mathrm{CH}_{3} \mathrm{NH}_{3} \mathrm{~Pb}\left(\mathrm{I}_{1-x} \mathrm{Br}_{x}\right)_{3}$ hybrid halide perovskite alloy. J. Phys. Chem. Lett. 7, 1083-1087 (2016).

69. Zhao, J. et al. Strained hybrid perovskite thin films and their impact on the intrinsic stability of perovskite solar cells. Sci. Adv. 3, eaao5616 (2017).

70. Tennyson, E. M., Doherty, T. A. S. \& Stranks, S. D. Heterogeneity at multiple length scales in halide perovskite semiconductors. Nat. Rev. Mater. 4, 573-587 (2019).

71. Draguta, S. et al. Rationalizing the light-induced phase separation of mixed halide organic-inorganic perovskites. Nat. Commun. 8, 200 (2017).

72. Li, Z. et al. Modulation of recombination zone position for quasi-two-dimensional blue perovskite light-emitting diodes with efficiency exceeding 5\%. Nat. Commun. 10, 1027 (2019). 
73. Vashishtha, P., Ng, M., Shivarudraiah, S. B. \& Halpert, J. E. High efficiency blue and green light-emitting diodes using Ruddlesden-Popper inorganic mixed halide perovskites with butylammonium interlayers. Chem. Mater. 31, 83-89 (2019).

74. Yantara, N. et al. Designing the perovskite structural landscape for efficient blue emission. ACS Energy Lett. 5, 1593-1600 (2020).

75. Si, J. et al. Efficient and high-color-purity light-emitting diodes based on in situ grown films of $\mathrm{CsPbX}_{3}(\mathrm{X}=\mathrm{Br}, \mathrm{I})$ nanoplates with controlled thicknesses. ACS Nano 11, 1110011107 (2017).

76. Tsai, H. et al. Stable light-emitting diodes using phase-pure Ruddlesden-Popper layered perovskites. Adv. Mater. 30, 1704217 (2018).

77. Tanaka, K. et al. Comparative study on the excitons in lead-halide-based perovskite-type crystals $\mathrm{CH}_{3} \mathrm{NH}_{3} \mathrm{PbBr}_{3} \mathrm{CH}_{3} \mathrm{NH}_{3} \mathrm{PbI}_{3}$. Solid State Commun. 127, 619-623 (2003).

78. Bekenstein, Y., Koscher, B. A., Eaton, S. W., Yang, P. \& Alivisatos, A. P. Highly luminescent colloidal nanoplates of perovskite cesium lead halide and their oriented assemblies. J. Am. Chem. Soc. 137, 16008-16011 (2015).

79. Bertolotti, F. et al. Crystal structure, morphology, and surface termination of cyanemissive, six-monolayers-thick $\mathrm{CsPbBr}_{3}$ nanoplatelets from $\mathrm{X}$-ray total scattering. ACS Nano 13, 14294-14307 (2019).

80. Jagielski, J. et al. Scalable photonic sources using two-dimensional lead halide perovskite superlattices. Nat. Commun. 11, 387 (2020). 
81. Bi, C. et al. Spontaneous self-assembly of cesium lead halide perovskite nanoplatelets into cuboid crystals with high intensity blue emission. Adv. Sci. 6, 1900462 (2019).

82. Linaburg, M. R., McClure, E. T., Majher, J. D. \& Woodward, P. M. $\mathrm{Cs}_{1-x} \mathrm{Rb}_{x} \mathrm{PbCl}_{3}$ and $\mathrm{Cs}_{1-x} \mathrm{Rb}_{x} \mathrm{PbBr}_{3}$ solid solutions: understanding octahedral tilting in lead halide perovskites. Chem. Mater. 29, 3507-3514 (2017).

83. Jiang, Y. et al. Spectra stable blue perovskite light-emitting diodes. Nat. Commun. 10, 1868 (2019).

84. van der Stam, W. et al. Highly emissive divalent-ion-doped colloidal $\mathrm{CsPb}_{1-x} \mathrm{M}_{x} \mathrm{Br}_{3}$ perovskite nanocrystals through cation exchange. J. Am. Chem. Soc. 139, 4087-4097 (2017).

85. Wang, H. et al. Trifluoroacetate induced small-grained $\mathrm{CsPbBr}_{3}$ perovskite films result in efficient and stable light-emitting devices. Nat. Commun. 10, 665 (2019).

86. Snaith, H. J. \& Hacke, P. Enabling reliability assessments of pre-commercial perovskite photovoltaics with lessons learned from industrial standards. Nat. Energy 3, 459-465 (2018).

87. Senocrate, A. et al. The nature of ion conduction in methylammonium lead iodide: a multimethod approach. Angew. Chem. Int. Ed. 56, 7755-7759 (2017).

88. Xu, M. et al. A transient-electroluminescence study on perovskite light-emitting diodes. Appl. Phys. Lett. 115, 041102 (2019). 
89. Sutanto, A. A. et al. In situ analysis reveals the role of $2 \mathrm{D}$ perovskite in preventing thermal-induced degradation in 2D/3D perovskite interfaces. Nano Lett. 20, 3992-3998 (2020).

90. Yu, J. C. et al. Improving the stability and performance of perovskite light-emitting diodes by thermal annealing treatment. Adv. Mater. 28, 6906-6913 (2016).

91. Zhao, L. et al. Redox chemistry dominates the degradation and decomposition of metal halide perovskite optoelectronic devices. ACS Energy Lett. 1, 595-602 (2016).

92. Babayigit, A., Ethirajan, A., Muller, M. \& Conings, B. Toxicity of organometal halide perovskite solar cells. Nat. Mater. 15, 247-251 (2016).

93. Ning, W. \& Gao, F. Structural and functional diversity in lead-free halide perovskite materials. Adv. Mater. 31, 1900326 (2019).

94. Lanzetta, L., Marin-Beloqui, J. M., Sanchez-Molina, I., Ding, D. \& Haque, S. A. Twodimensional organic tin halide perovskites with tunable visible emission and their use in light-emitting devices. ACS Energy Lett. 2, 1662-1668 (2017).

95. Ma, Z. et al. Electrically-driven violet light-emitting devices based on highly stable leadfree perovskite $\mathrm{Cs}_{3} \mathrm{Sb}_{2} \mathrm{Br}_{9}$ quantum dots. ACS Energy Lett. 5, 385-394 (2020).

96. Jun, T. et al. Lead-free highly efficient blue-emitting $\mathrm{Cs}_{3} \mathrm{Cu}_{2} \mathrm{I}_{5}$ with $0 \mathrm{D}$ electronic structure. Adv. Mater. 30, 1804547 (2018).

97. Lu, S. et al. Accelerated discovery of stable lead-free hybrid organic-inorganic perovskites via machine learning. Nat. Commun. 9, 3405 (2018). 
98. Gong, X. et al. Highly efficient quantum dot near-infrared light-emitting diodes. Nat. Photon. 10, 253-257 (2016).

99. Vasilopoulou, M. et al. Efficient colloidal quantum dot light-emitting diodes operating in the second near-infrared biological window. Nat. Photon. 14, 50-56 (2020).

100. Mao, J. et al. All-perovskite emission architecture for white light-emitting diodes. ACS Nano 12, 10486-10492 (2018).

101. Li, S., Luo, J., Liu, J. \& Tang, J. Self-trapped excitons in all-inorganic halide perovskites: fundamentals, status, and potential applications. J. Phys. Chem. Lett. 10, 1999-2007 (2019).

102. Zhou, N. et al. Perovskite nanowire-block copolymer composites with digitally programmable polarization anisotropy. Sci. Adv. 5, eaav8141 (2019).

103. Bade, S. G. R. et al. Fully printed halide perovskite light-emitting diodes with silver nanowire electrodes. ACS Nano 10, 1795-1801 (2016).

104. Zhao, L. et al. Influence of bulky organo-ammonium halide additive choice on the flexibility and efficiency of perovskite light-emitting device. Adv. Funct. Mater. 28, 1802060 (2018).

105. Chou, S.-Y. et al. Transparent perovskite light-emitting touch-responsive device. ACS Nano 11, 11368-11375 (2017).

106. Xing, G. et al. Low-temperature solution-processed wavelength-tunable perovskites for lasing. Nat. Mater. 13, 476-480 (2014). 
107. Gunnarsson, W. B. \& Rand, B. P. Electrically driven lasing in metal halide perovskites: challenges and outlook. APL Mater. 8, 030902 (2020).

108. Kim, H. et al. Hybrid perovskite light emitting diodes under intense electrical excitation. Nat. Commun. 9, 4893 (2018).

109. Jia, Y. et al. Diode-pumped organo-lead halide perovskite lasing in a metal-clad distributed feedback resonator. Nano Lett. 16, 4624-4629 (2016).

110. Zhao, L. et al. Thermal management enables bright and stable perovskite light-emitting diodes. Adv. Mater. 32, 2000752 (2020).

\section{Acknowledgements}

We thank O. Inganäs, J. Qin, and N.K. Kumawat for insightful discussions. We acknowledge the financial support from the ERC Starting Grant (No. 717026), the Swedish Energy Agency Energimyndigheten (No. 48758-1), the Swedish Foundation for International Cooperation in Research and Higher Education (No. CH2018-7736), and the Swedish Government Strategic Research Area in Materials Science on Functional Materials at Linköping University (Faculty Grant SFO-Mat-LiU No. 2009-00971). R.H.F. acknowledges support from the EPSRC. J.W. acknowledges financial support from the Joint Research Program between China and European Union (2016YFE0112000). Y.J. acknowledges support from the National Key Research and Development Program of China (2016YFB0401600) and the National Natural Science Foundation of China (21975220, 91833303, 91733302 and 51911530155). X.-K.L. is a Marie Skłodowska-Curie Fellow (No. 798861). F.G. is a Wallenberg Academy Fellow.

\section{Additional information}

Reprints and permissions information is available online at www.nature.com/reprints.

Correspondence and requests for materials should be addressed to F.G.

\section{Competing financial interests}


The authors declare no competing financial interests. 
a

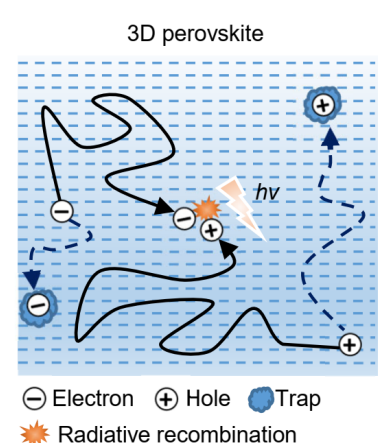

b

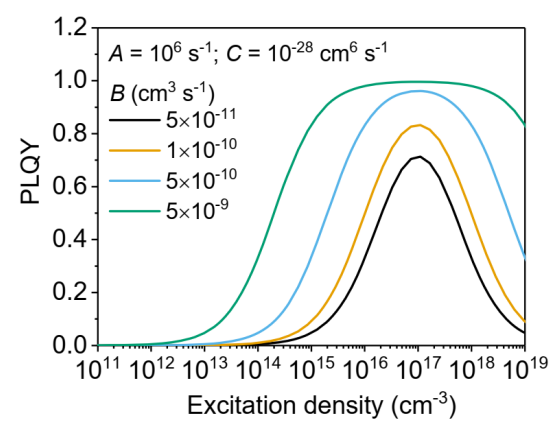

c

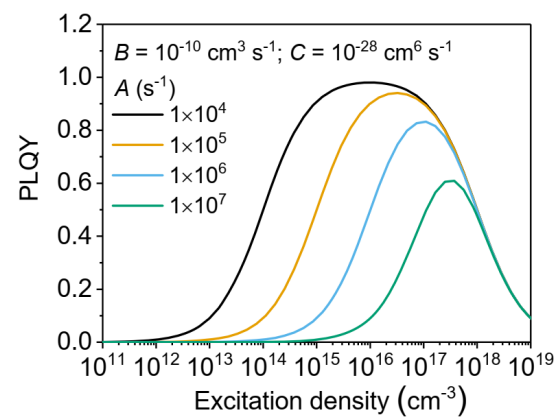

Quantum-confined perovskite
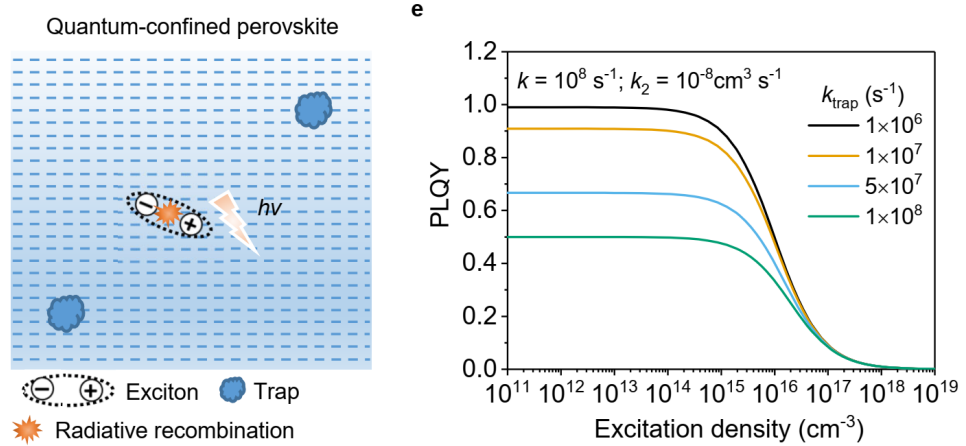

Figure 1 | Charge-carrier recombination kinetics. a, A schematic illustration of chargecarrier recombination in 3D perovskites. b,c, Related PLQY-excitation density characteristics with (b) varying bimolecular recombination coefficients and (c) varying nonradiative monomolecular recombination coefficients. d, A schematic illustration of charge-carrier recombination in quantum-confined perovskites. e, Related PLQY-excitation density plots with varying trap-assisted nonradiative recombination rate constants. Figure adapted with permission from: a,d, ref. ${ }^{17}$, under a Creative Commons licence (http://creativecommons.org/licenses/by/4.0/). 


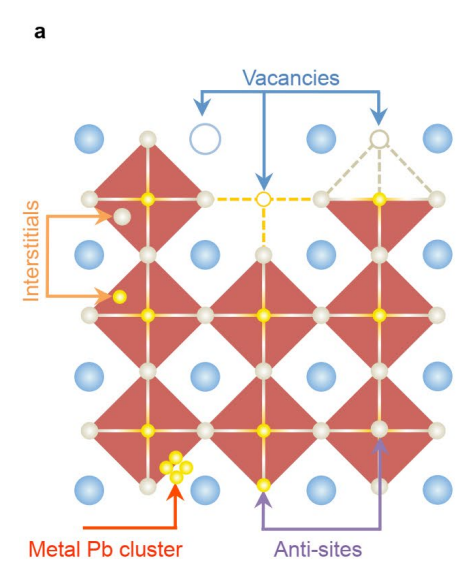

$\mathrm{A}^{+} \odot \mathrm{B}^{2+} \odot \mathrm{X}^{-}$

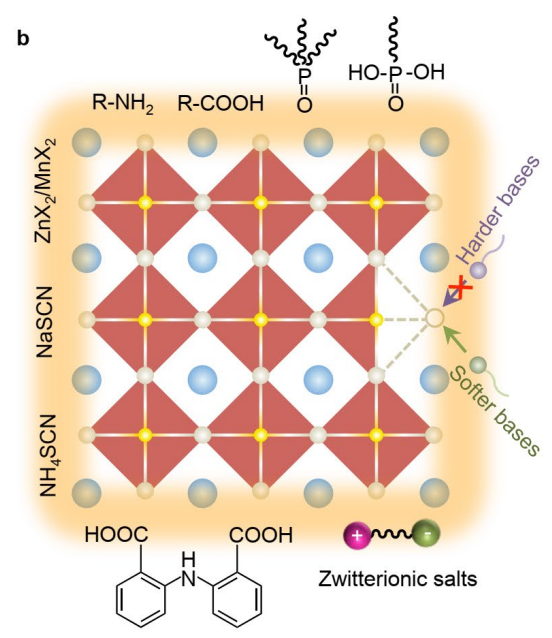

Figure 2 | Defects in perovskites and defect passivation. a, Point defects of perovskites including vacancies, interstitials, anti-sites and metal $\mathrm{Pb}$ clusters. $\mathbf{b}$, Categories of passivating ligands for colloidal PNCs; the right side demonstrates the hard-soft acids-bases theory that was used in ref. ${ }^{32}$ to predict the passivation effects of Lewis base ligands for $\mathrm{CsPbX}_{3}$ PNCs. c, $\mathrm{An}$ illustration demonstrating the modulation of passivation effects by rational molecular design reported in ref. ${ }^{6}$. 

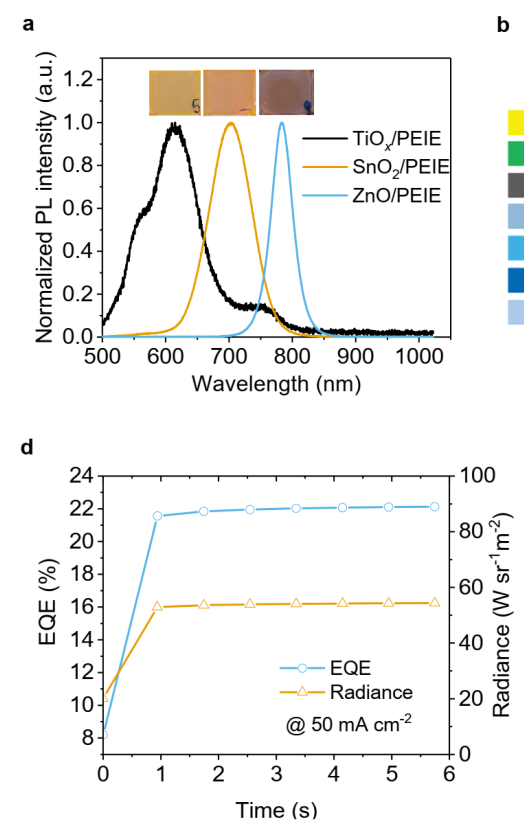
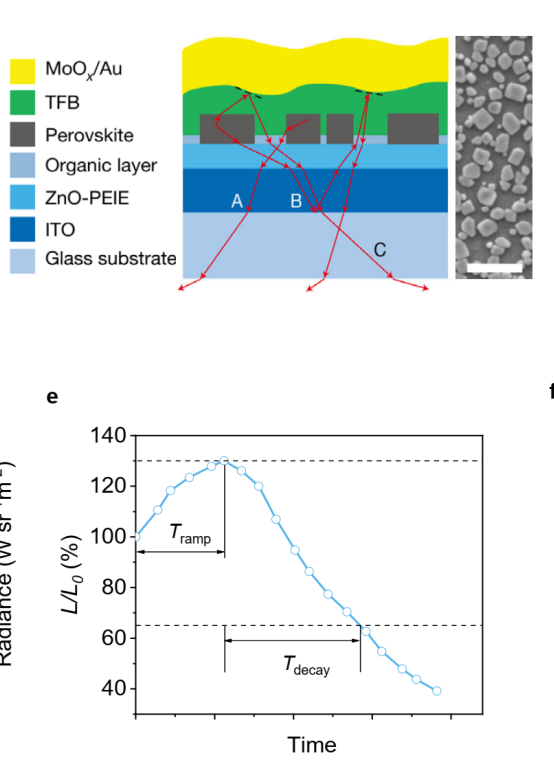
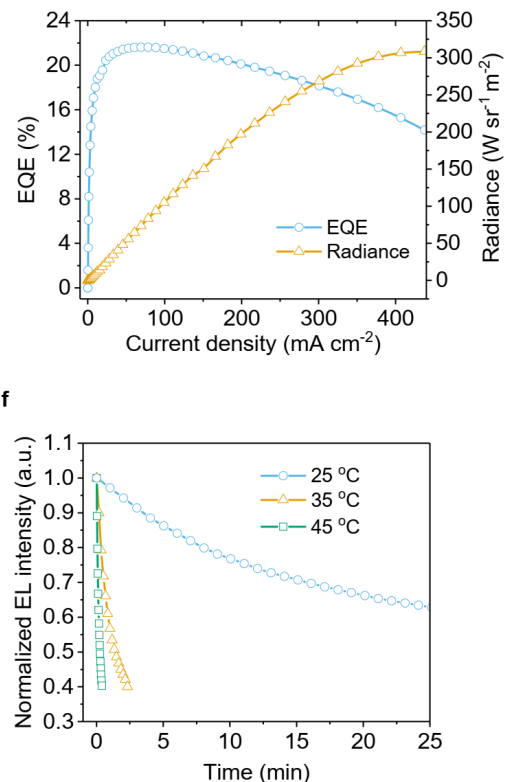

Figure 3 | Device aspects of PeLEDs. a, Normalized PL spectra and photographs (inset) of perovskite films on $\mathrm{TiO}_{x} / \mathrm{PEIE}, \mathrm{SnO}_{2} / \mathrm{PEIE}$ and $\mathrm{ZnO} / \mathrm{PEIE}$ substrates prepared from the same precursor solution, indicating that the interlayers can significantly affect the properties of the perovskite layer; PEIE is polyethylenimine ethoxylated. $\mathbf{b}$, An illustration (left panel) of a device with submicrometre-sized perovskite platelets, where rays $\mathrm{A}, \mathrm{B}$ and $\mathrm{C}$ represent light, otherwise trapped in devices with a continuous emitting layer, that can be extracted by the submicrometre structure; TFB is poly(9,9-dioctyl-fluorene-co-N-(4-butylphenyl)diphenylamine). Top-view scanning electronic microscopic (SEM) image (right panel) of the perovskite film, with a scale bar of $1 \mu \mathrm{m}$. c,d, Examples of the commonly used EQE- and radiance-current density curve (c), and stabilized EQE and radiance at a constant current density for the same device (d). e, A schematic operational stability curve of PeLEDs with an obvious increase in the luminance during the measurement. $T_{\text {ramp }}$, the needed time for the initial luminance to increase to the peak value; $T_{\text {decay }}$, the needed time for the peak luminance to drop to $50 \%$ of the value. f, Operational stability of an $\mathrm{FAPbI}_{3}$ PeLED measured at various temperatures at 100 $\mathrm{mA} \mathrm{cm}{ }^{-2}$. Figure adapted with permission from: a, ref. ${ }^{47}$, under a Creative Commons licence (http://creativecommons.org/licenses/by/4.0/); b, ref. ${ }^{8}$, Springer Nature Ltd; c, ref. ${ }^{6}$, Springer 
Nature Ltd; f, ref. ${ }^{63}$, under a Creative Commons licence (http://creativecommons.org/licenses/by/4.0/).

a
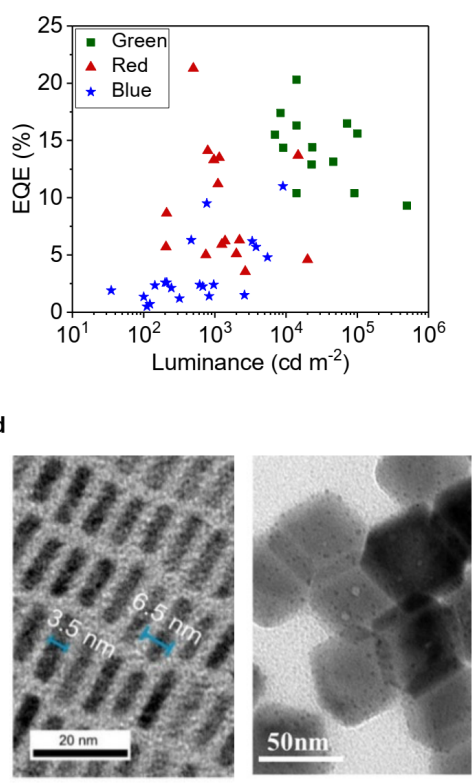

b

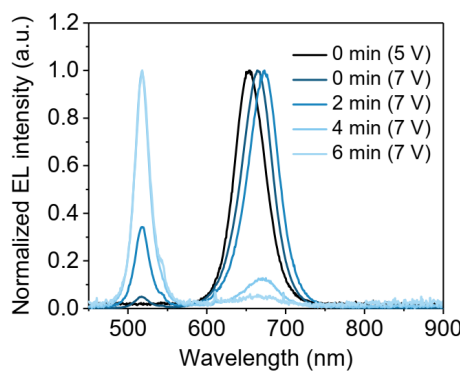

e

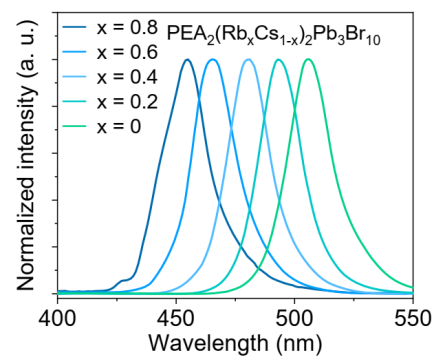

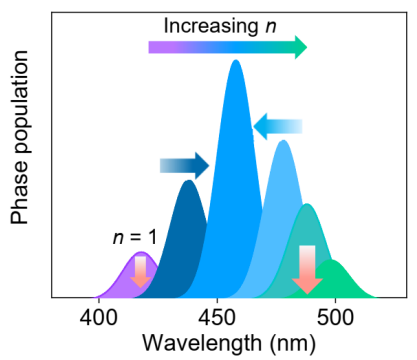

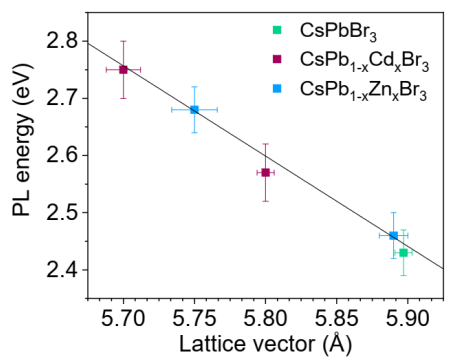

Figure 4 | Challenges in high-performance blue and red PeLEDs. a, Summary of EQE and luminance of state-of-the-art blue, green and red PeLEDs; the data used for this figure are listed in Supplementary Table 1. b, Normalized electroluminescence (EL) spectra of a $\mathrm{CsPb}(\mathrm{Br} / \mathrm{I})_{3}$ based PeLED during operation. c, A schematic diagram of precise dimensional control in mixed dimensional perovskites towards narrow dimensional distribution and Rec. 2020 primary blue emission. d, Low-resolution transmission electron microscopic (TEM) image (left panel) of stacked nanoplatelets (thicknesses are around 3.5 and $6.5 \mathrm{~nm}$ without and with the ligand shell, respectively) and TEM image (right panel) of $\mathrm{CsPbBr}_{3}$ cuboid $\mathrm{NCs}$ assembled from nanoplatelets. e, Normalized PL spectra of $\mathrm{PEA}_{2}\left(\mathrm{Rb}_{x} \mathrm{Cs}_{1-x}\right)_{2} \mathrm{~Pb}_{3} \mathrm{Br}_{10}$ perovskites, demonstrating the effect of $\mathrm{Rb}^{+}$incorporation on the bandgap. $\mathbf{f}, \mathrm{PL}$ energy as a function of lattice vector in doped $\mathrm{CsPb}_{1-x} \mathrm{M}_{x} \mathrm{Br}_{3}(\mathrm{M}=\mathrm{Cd}$ and $\mathrm{Zn}) \mathrm{NCs}$ and their parent $\mathrm{CsPbBr}_{3} \mathrm{NCs}$, indicating that impurity metal ions in the perovskite lattice can tune the bandgap of perovskite NCs. Figure adapted with permission from: b, ref. ${ }^{66}$, American Chemical Society; c, ref. ${ }^{74}$, American 
Chemical Society; d (left panel), ref. ${ }^{79}$, American Chemical Society; d (right panel), ref. ${ }^{81}$, under a Creative Commons licence (http://creativecommons.org/licenses/by/4.0/); e, ref. ${ }^{83}$, under a Creative Commons licence; f, ref. ${ }^{84}$, American Chemical Society.
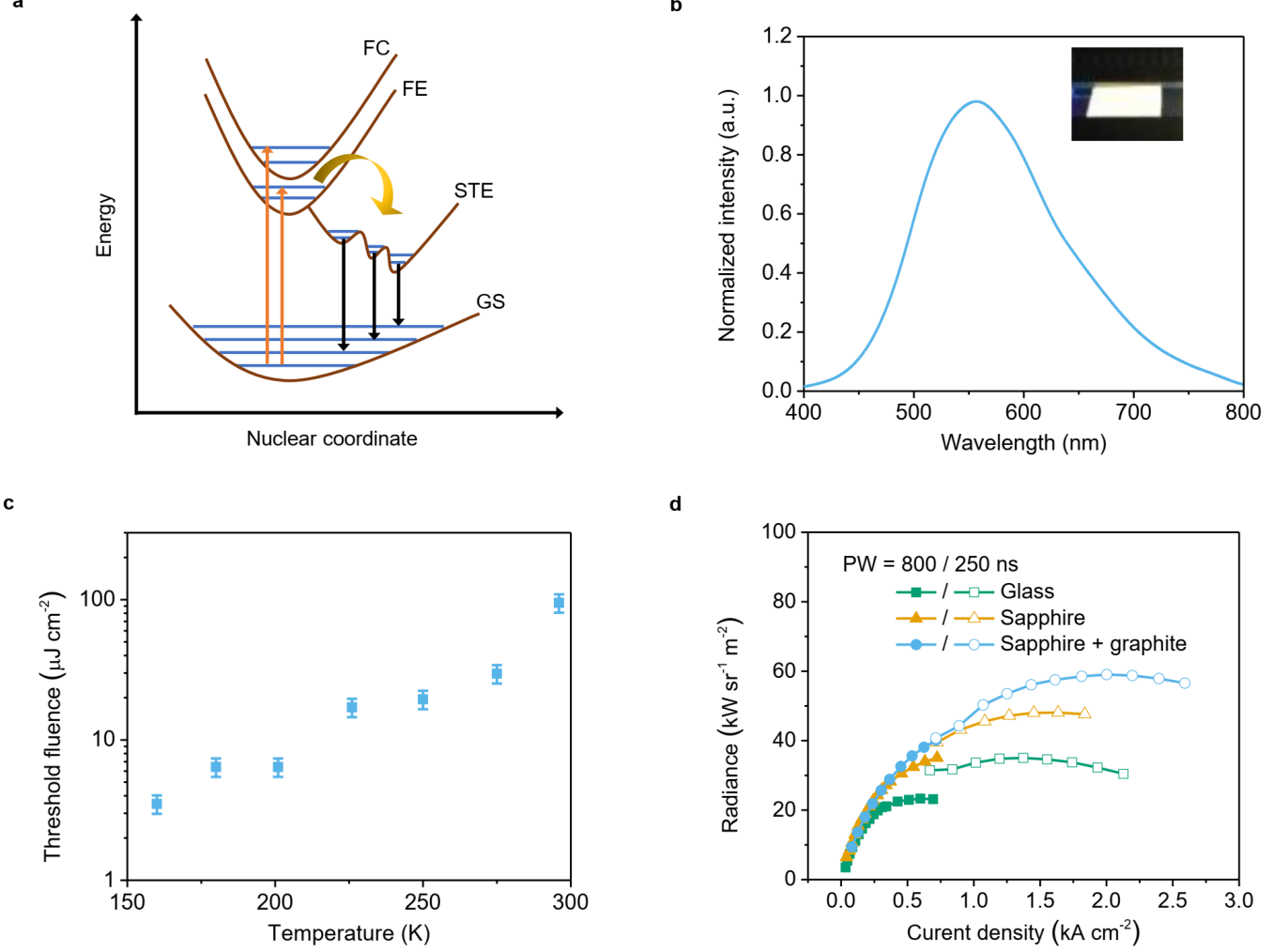

Figure 5 | Prospects of perovskites in white PeLEDs and electrically pumped lasers. a, A schematic illustration of STE emission; FC, free carrier state; FE, free exciton state; STE, selftrapped exciton state; GS, ground state. b, Normalized PL spectrum of $\mathrm{Cs}_{2} \mathrm{Ag}_{0.60} \mathrm{Na}_{0.40} \mathrm{InCl}_{6}$ measured at $293 \mathrm{~K}$; the inset shows a 300-nm-thick $\mathrm{Cs}_{2} \mathrm{Ag}_{0.60} \mathrm{Na}_{0.40} \mathrm{InCl}_{6}$ film under 254-nm ultraviolet illumination. c, Pump threshold fluence as a function of temperature for $\mathrm{MAPbI}_{3}$. d, Radiance-current density curves of pulse driven PeLEDs on glass or sapphire substrates, with or without a graphite heat spreader and copper heat sink; PW, pulse width. Figure adapted with permission from: b, ref. ${ }^{12}$, Springer Nature; c, ref. ${ }^{109}$, American Chemical Society; d, ref. ${ }^{110}$, Wiley. 

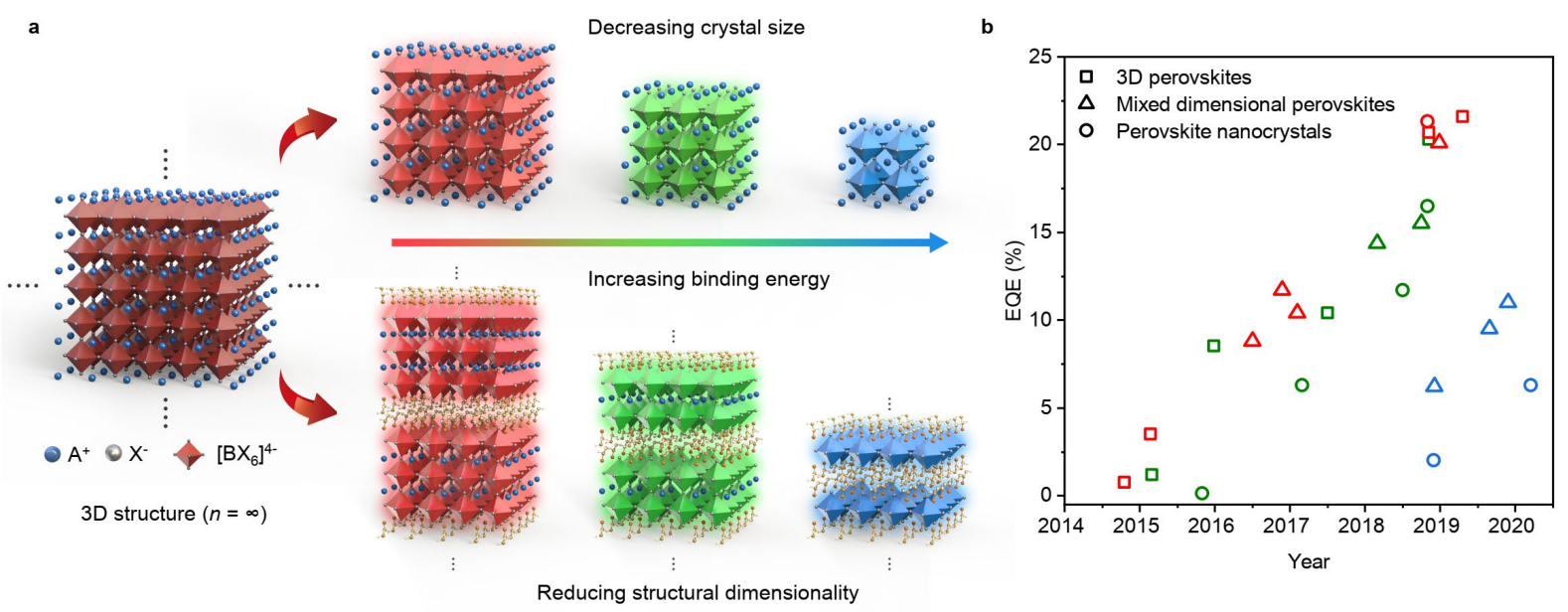

Figure B1 | The development of perovskite emitters and PeLEDs. a, Material diversity of metal halide perovskite emitters. Beyond 3D structures, nanostructures (including quantumconfined structures) can be obtained by decreasing the crystal size or reducing the structural dimensionality, resulting in increased electron-hole (exciton) binding energy. b, Development of PeLEDs with different emitters and different light emissions; the red, green, and blue symbols denote red/near-infrared, green and blue PeLEDs, respectively. The data used for this figure are listed in Supplementary Table 1. 


\section{Box 1. The development of perovskite emitters and PeLEDs.}

Metal halide perovskites refer to a large family of crystalline materials with structures similar to that of the natural mineral calcium titanate, which was discovered by Gustav Rose in 1839 and named after Russian mineralogist Lev Perovski. Metal halide perovskites can be described with a general chemical formula of $\mathrm{ABX}_{3}$, where $\mathrm{A}$ is a monovalent cation, $\mathrm{B}$ is a divalent metal cation, and $\mathrm{X}$ is a halogen anion that bonds with metal cations, forming corner-sharing $\left[\mathrm{BX}_{6}\right]^{4-}$ octahedra cavities embedding the A cations. Limited by the Goldschmidt tolerance factor of the perovskite phase, A-site cations in such three-dimensional (3D) structures are mostly cesium $\left(\mathrm{Cs}^{+}\right)$, methylammonium $\left(\mathrm{MA}, \mathrm{CH}_{3} \mathrm{NH}_{3}{ }^{+}\right.$) or formamidinium $\left(\mathrm{FA}, \mathrm{CH}\left(\mathrm{NH}_{2}\right)_{2}{ }^{+}\right.$) cations with suitable sizes.

From the 3D structures, nanostructures or even quantum-confined structures can be derived by decreasing the crystal size or reducing the structural dimensionality (Fig. B1,a). Using colloidal approaches, perovskite nanocrystals have been successfully synthesized with different sizes and shapes, including nanocubes, nanoplatelets, nanosheets, nanowires and quantum $\operatorname{dots}^{13}$. Alternatively, reduced structural dimensionality - namely two-dimensional (2D) or quasi-2D layered perovskites such as Ruddlesden-Popper and Dion-Jacobson phases - has been obtained by incorporating large-sized organic cations into the perovskite frameworks. These materials share a formula of $\mathrm{L}_{m} \mathrm{~A}_{n-1} \mathrm{~B}_{n} \mathrm{X}_{3 n+1}$, where $\mathrm{L}$ is typically a large-sized monovalent $(m$ $=2$ ) or divalent $(m=1)$ cation, and $n$ is the number of inorganic octahedra sheets between the large-sized organic spacers, with the case of $n=\infty$ representing the 3D structures. The large compositional and dimensional diversity obtained by combining different cations and anions in the crystal structures offers wide tunability of the optoelectronic properties of perovskite emitters.

Early reports of PeLEDs date back to the 1990s (refs ${ }^{3,4}$ ). The devices at that time either operated at cryogenic temperatures or emitted broad electroluminescence (EL) from the organic 
moieties in the material structure, attracting limited attention. PeLEDs demonstrating roomtemperature EL with narrow emission were developed in $2014^{5}$, setting a new start for the evolution of this research field (Fig. B1,b). Early-stage development of PeLEDs mostly involved 3D perovskites, with a focus on uniform film morphology and reduced grain sizes ${ }^{20,21}$. More recently, it was found that discontinuous perovskite films could increase light outcoupling and improve efficiency, as long as leakage currents could be effectively suppressed ${ }^{8}$. Along with the development of 3D perovskite emitters, perovskite films made of mixtures of 2D/quasi-2D/3D phases (hereafter referred to as mixed dimensional perovskites) have also attracted significant attention for high-efficiency PeLEDs ${ }^{26,27}$. Such perovskites demonstrate much enhanced confinement of charge carriers as well as effective radiative recombination within the lowest-energy perovskite phase.

In addition to the polycrystalline thin films deposited directly from precursor solutions, assemblies of colloidal PNCs have also been used in PeLEDs. Since the PNC-based PeLEDs reported in $2015^{48}$, intensive efforts have been dedicated to optimizing material compositions (for instance through A-site substitution and metal ion doping), purification protocols and surface ligands ${ }^{13}$. These efforts have led to controllable luminescent properties of PNCs and rapid performance improvement of the ensuing PNC-based PeLEDs ${ }^{9,13,36}$.

In all these different perovskite emitters, it is of critical importance to effectively eliminate non-radiative recombination pathways for achieving high-performance PeLEDs. A wide range of effective strategies have been developed, including quasi-core-shell structures ${ }^{7}$, perovskitepolymer heterostructures ${ }^{10}$, and rational hydrogen bonding manupiluation ${ }^{6}$. These efforts have pushed the EQEs of PeLEDs over 20\%, approaching those of organic LEDs (OLEDs) and conventional QD-based LEDs (QLEDs). 